\title{
Memristor State-Space Embedding
}

\author{
${ }^{1,2}$ Wael Dghais, ${ }^{1,2}$ Luis Nero Alves, ${ }^{1}$ Joana Catarina Mendes, ${ }^{1,2}$ Jonathan Rodriguez, ${ }^{1,2}$ José Carlos Pedro \\ ${ }^{1}$ Dept. de Electrónica, Telecomunicações e Informática, Universidade de Aveiro, Portugal \\ ${ }^{2}$ Instituto de Telecomunicações, Aveiro, Portugal \\ waeldghais@ua.pt, nero@av.it.pt, joanacatarina.mendes@av.it.pt, jonathan@av.it.pt, jcpedro@ua.pt
}

\begin{abstract}
This paper presents a procedure for the determination of the dimensionality of the state space of a memristive device. The state space dimensionality of a device corresponds to the minimum number of time delayed corresponds to the minimum number of time delayed
values/derivatives of the voltage and current required to values/derivatives of the voltage and current required to
represent the device dynamics for a specified set of inputs. The algorithm is based on the observed time domain voltage-current (i.e. input-output) data which is obtained by measurement. The determination of the state space dimensionality is important to achieve a single-alued input-output mapping between the device outputs as a function of the embedding state variables. In this paper, this will be accomplished using an embedding technique, based on the false nearest neighbor principle.
\end{abstract}

Keywords-memristor device, nonlinear behavioral modelling, false nearest neighbour, state-space embedding.

\section{INTRODUCTION}

The finger prints of a memristive device (MD) are derived from the interpretation of the observed time parameterized output trajectory. This is normally constructed using inputoutput (I/O) data recorded under sinusoidal excitation at various amplitudes and frequencies [1], [2]. The time-domain behavior of the MD can be described by the canonical state equations of the form:

$$
\left\{\begin{array}{l}
\frac{d \vec{w}(t)}{d t}=\vec{f}(\vec{w}(t), v(t)) \\
i(t)=h(\vec{w}(t)) \cdot v(t)
\end{array}\right.
$$

where $v(t)$ and $i(t)$ are the observable applied voltage and output current, respectively, while $\vec{w}(t)$ is the unobservable state vector. The loop observed in the $i(t)-v(t)$ trajectory plane is the an evidence of the memory effects exhibited by these devices. Equation (1) is normally employed for voltage controlled devices. It comprises the state equation and the input-output mapping governing the dynamics of the MD. Moreover, current-controlled devices are defined in a similar manner [1].

The number of state (e.g. embedding) variables describes the order of the system. The observable output current $i(t)$ can be predicted for every time $t$ when the state equations and the observable input signal are known or determined from observable data. This solution describes a time-parameterized path, or trajectory, in the multidimensional space of the state vector according to the frequency range and the amplitude variation of the input excitation signal.

When modelling a nonlinear device, such as an MD, two approaches are normally available: physics-based and black box, (i.e. behavioral) modelling approaches. Firstly, physicsbased modelling, employs a theoretical analysis of the physical mechanisms governing the device structure and materials to describe the MD's switching dynamic behavior. This approach determines the $h(\cdot)$ and $f(\cdot)$ functions in (1), and the state space vector $\vec{w}$,-based on complex physical formulation which may not provide a general and accurate representation of the experimental MD behavior due to some - simplifying assumptions (for instance constraining the underlying physical mechanisms for conduction)applied for a specific MD structure and materials (i.e. electrodes and resistive materials) during the model's derivation that can plague its use and implementation [1]. However, black-Black box modeling, deals directly with the observable quantities obtained from a specified set of input stimulus. The performance of an input-output black-box model's generation relies on the accurate determination of the dimension of the device's embedding variables and then the efficient parameterization of the nonlinear functional mapping between the device's identified state-space embedding dimension and its output [3]

Therefore, the determination of an accurate approximation of the sate-space vector, $\vec{w}$, based on the I/O recorded signals, will lead to - a reduced-order representation of the MD's physical dynamics while preserving a good behavioral model's prediction's accuracy. Up until now, most of the effort towards a universal model suitable for MDs has been focused in a physics based approach, biased by the Hewlett Packard (HP) team reported results [4]. It has been ascertained that the accuracy of these models is questionable for some applications [5], thus requiring better solutions and research effort. Given the wide range of materials and devices exhibiting memristive effects, it seems adequate to concentrate in a single modeling approach. Black box modeling is in this sense fitted to this quest, since it provides uniform procedures to assist the task of model development.

The first task in behavioral modelling is to find the embedding variables dimensionality of the device based on the observable quantities. This is known as embedding or statespace reconstruction. This step consists in determining the level of the MD's dynamics, which corresponds to the orders of voltage and current time delayed values and/or derivatives of the observables to retrieve the necessary internal state embedding variables of the model or their analogs. 


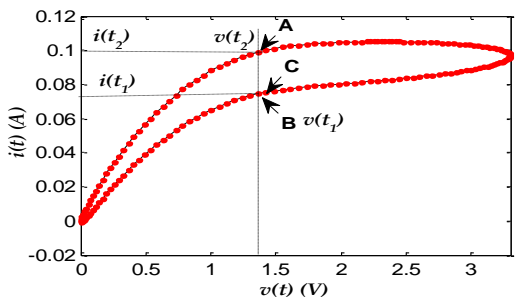

Fig. 1. The $i(t)-v(t)$ trajectory of PMOS transistor $\left(\mathrm{V}_{g s}=0 \mathrm{~V}, \mathrm{~V}_{d s}=\right.$ $A \cos (2 \pi f t), f=600 \mathrm{MHz})$

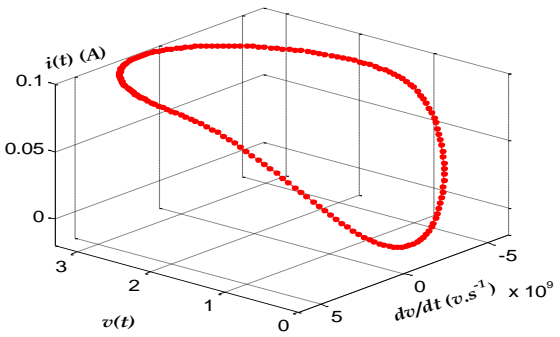

Fig. 2. The transistor's output current $i(t)$ is a single-valued function of the new embedding $\{v(t), d v(t) / d t\}$ space.

In this work we suggest to determine the appropriate embedding variables dimension for a sinusoidal driven MD by means of the false nearest neighbors (FNN) technique [6]. This

allows to find the minimum number of embedding variables required for the model and also to assist in model order reduction.

The rest of the paper is organized as follows. Section II is an illustration of the use of the FNN technique for space embedding dimension applied to the output current of a PMOS transistor, while Section III details the numerical results of the application of the FNN algorithm to an MD. Finally, conclusions are drawn in Section IV.

\section{STATE-SPACE EMBEDDING DIMENSION}

\section{A. Waveform and Memory}

Given a time series of the observable I/O signal waveforms, the state space model can be constructed using the embedding process. To illustrate the idea, the time-domain waveform of a PMOS transistor's output current $i(t)$ is plotted as a function of the sinusoidal drain-source voltage excitation $v(t)$ as shown in Fig. 1. The loop of the transistor's output current is a clear indication that $i(t)$ is not a single-valued function of $v(t)$. In fact, the transistor's output current can be seen as a parallel connection of a nonlinear current source (I-V) and a charge source $(\mathrm{Q}-\mathrm{V})$. For instance, by selecting two different time instants at the points $\mathrm{A}$ and $\mathrm{B}$, where the input satisfies $v\left(t_{1}\right)=v\left(t_{2}\right)$, the output current is different $i\left(t_{1}\right) \neq i\left(t_{2}\right)$ as illustrated. This demonstrates that the output current is not a single-valued, i.e. memoryless, or static, function of the input voltage. The points $\mathrm{A}$ and $\mathrm{B}$ are known as "false nearest neighbors" because they are close in the input space, but are from temporally distant events on the response curve or the output space. The point $\mathrm{C}$, which is close to point $\mathrm{B}$ on the response curve, is a true nearest neighbor of B [6].

After expanding the number of embedding dimensions by sampling $i(t)$ in the new embedding $\{v(t), d v(t) / d t\}$ space the response curve appears unfolded into a single-valued trajectory. Fig. 2 shows that at any time instant $i(t)$ can be expressed as a nonlinear single-valued, or memoryless, function of the two variables $i(t)=f(v(t), d v(t) / d t)$.

\section{B. FNN algorithm}

The method of false nearest neighbors (FNN) was developed by Kennel, Brown and Abarbanel [6] specifically to determine the minimum embedding dimension, i.e. the numbe of time-delayed observations, necessary to model the dynami behavior of autonomous chaotic systems. This method was extended to approximate the proper regression for $\mathrm{I} / \mathrm{O}$ dynamic processes, by modifying the regression vector and the search algorithm [7]. The regression vector can contain discrete timedelayed versions (2) or successive higher derivative (3) of both the input and the output signals.

$$
Z_{k, l}(t)=[v(t), v(t-\tau), \ldots v(t-(k-1) \tau), i(t
$$$$
-\tau), \ldots, i(t-(l-1) \tau)]
$$

$$
\begin{gathered}
Z_{k, l}(t)=\left[v(t), ., \mathrm{d} v(t) / d t, \frac{d^{(k-1)} v(t)}{d t^{(k-1)}}\right. \\
\left., \frac{\mathrm{d} i(t)}{d t}, \ldots, \frac{d^{(l-1)} i(t)}{d t^{(l-1)}}\right]
\end{gathered}
$$

where $\tau$ is the time delay, $k$ and $l$, are the current and voltage embedding dimensions, respectively. The time delay, $\tau$, should be chosen so that the I/O signals are de-correlated from their delayed versions. For instance, the autocorrelation function can provide the adequate delay $\tau=T / 4$ for periodic sinusoidallike signals, this assures that each signal is de-correlated from its previous delayed versions. Moreover, for nonlinear dynamic system analysis, excited by broadband input signals (fo instance multisines), the method of mutual information from information theory can be employed to determine the appropriate delay $\tau$ [8]. The discrete and/or continuous time regression vector can be mixed or used independently for determining the embedding dimension. However, the discrete regression vector (2) is more general because the derivative involves a time delay version of the signal $(d v(t) / d t \cong$ $\left(v(t)-v\left(t-T_{s}\right)\right) / T_{s}$, where $T_{s}$ is the sampling time).

In order to determine the proper dimension of $Z_{k, l}(t)$, the percentage of FNN must be evaluated for different numbers of delayed version of the $\mathrm{I} / \mathrm{O}$ signals. This is done by finding/searching for the nearest neighbors in terms of the Euclidean distance to another time instant $h$, that must diffe from $t$ by, at least, a time sample, -in the I/O sampling space, $Z_{k, l}(h)$, such that the distance $d$ is minimized [4]

$$
d=\left\|Z_{k, l}(t)-Z_{k, l}(h)\right\|_{2}
$$




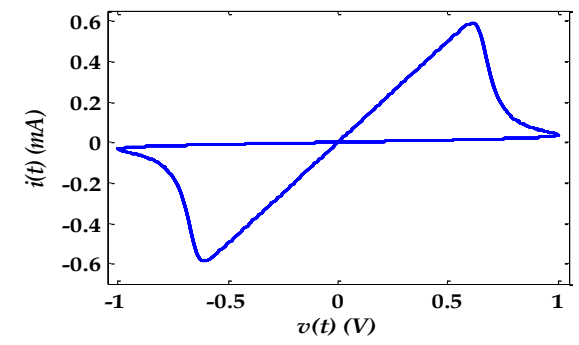

Fig. 3. Nonlinear looping effect in the $i(t)-v(t)$ plane trajectory of the nemristor's admittance.

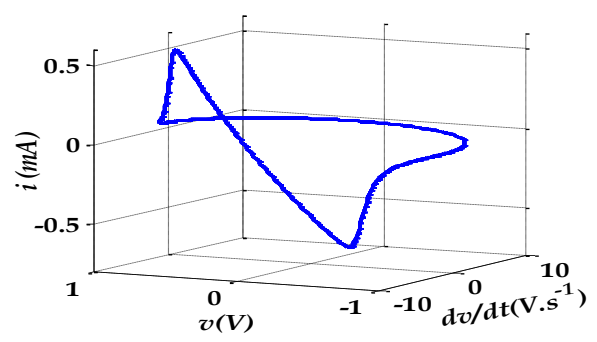

Fig. 4. Trajectory of $i(t)$ as a function of $v(t)$ and $d v(t) / d t$.

A test is defined to determine whether the neighbors have future outputs that are distinct, that is, whether neighbors are true or false. For this purpose, the ratio in (5) determines whether the distance between future outputs, $i(t)$ and $i(h)$ is significantly larger than the distance between discrete/continuous time regression vectors, $Z_{k, l}(t)$ and $Z_{k, l}(h)$ that are close in the regressed space [7]

$$
\frac{|i(t)-i(h)|}{\left\|Z_{k, l}(t)-Z_{k, l}(h)\right\|_{2}} \leq R
$$

These steps will be repeated for all times $t$ and $h$ in the data set while calculating the percentage of the time series points that have false nearest neighbors. The regression vector, $Z_{k, l}(t)$, will be extended (increasing $l$ or $m$ ) to include more delayed versions of the input-output space until the percentage of FNN drops to an acceptably small number. It is worth noting that, before this two-dimensional search algorithm, a normalization step of all the $\mathrm{I} / \mathrm{O}$ signals and/or their derivatives is performed to fall in the range of $[-1,1]$. This will result in more accurate and better convergence of the FNN algorithm. As the embedding variables are incremented, the state space is unfolded into a higher number of dimensions till it reaches a dimension at which the output response or the current curve will unfold into a single-valued trajectory without looping effect where each point will be a true nearest neighbor.

III. Memristor State-Space EMBEDDing DimEnsion

To illustrate the procedure for a MD, the input-output data was generated using an MD described by the HP model with Prodromakis window function, with $A=1$ and $p=5$ [9]. The

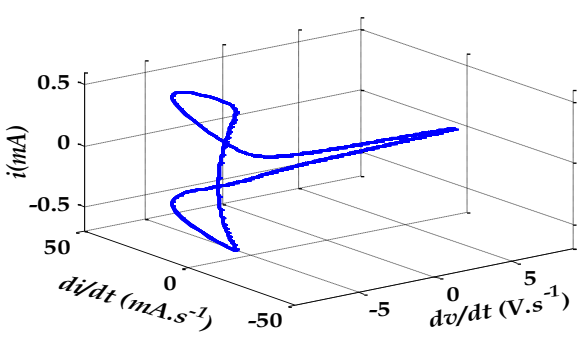

Fig. 5. Trajectory of $i(t)$ as a function of $d i(t) / d t$ and $d v(t) / d t$.

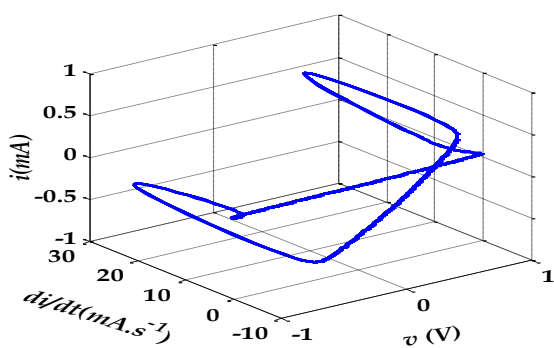

Fig. 6. Trajectory of $i(t)$ as a function of $d i(t) / d t$ and $v(t)$.

simulation was performed with Matlab using the ode15s differential solver. The data set contains the steady state response of the device, when excited with an input sinusoid of frequency $f=1.32 \mathrm{~Hz}$ and amplitude of $1.0 \mathrm{~V}$. The device parameters used were: $R_{o n}=1 \mathrm{k} \Omega, R_{\text {off }}=100 \mathrm{k} \Omega, \eta=1, \mu=1 \mathrm{E}-14$ $\mathrm{m}^{2} \mathrm{~s}^{-1} \mathrm{~V}^{-1}, D=10 \mathrm{~nm}$, and $w_{o}=5 \mathrm{~nm}$. The input space of the regression vector is formed by the successive derivatives of $v(t)$ as shown in (6):

$$
\left\{\begin{array}{l}
v(t)=a \cos (\omega t) \\
\dot{v}(t)=-a \omega \sin (\omega t) \\
\ddot{v}(t)=-a \omega^{2} \cos (\omega t)=-\omega^{2} v(t) \\
\dddot{v}(t)=a \omega^{3} \sin (\omega t)=-\omega^{2} \dot{v}(t)
\end{array}\right.
$$

It is noticeable that the input signal and its first derivative are representative of all the dynamic information present in the input space of the $Z_{k, l}(t)$ since the second derivative (i.e. $\ddot{v}(t)$ ) and all the higher order ones can be linearly related to either $v(t)$ or $\dot{v}(t)$, which are the only ones orthogonal over the period $T$. This means that $v(t)$ and $\dot{v}(t)$ are sufficient to incorporate all the necessary input space dynamics, into the MD model. Therefore, adding more derivatives of $v(t)$ in the input space when the MD is driven by a sinusoidal-like signal will not add any further information that will help to embed the input space.

Consequently, the delayed versions or the derivatives of output space - current information - should be added to the input space to obtain a single-valued mapping function between the state-space and the output current. The nonlinear 


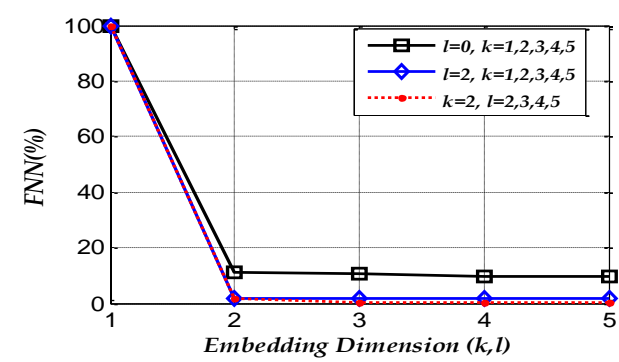

Fig. 7. The percentage of FNN vs. the input space ( $k$ ) (solid line) and output space $(I)$ (dashed line) embedding dimension with lists of selected initial state variables.

TABLE I. FNN RESULTS FOR DIFFERENT DIMENSION OF THE I/O EMBEDDING VARIABLES OF THE MD.

\begin{tabular}{|c|c|c|c|c|}
\hline \multicolumn{2}{|c|}{ Embedding Dimension } & \multicolumn{2}{|c|}{ State -Variable } & $\begin{array}{c}\text { FNN } \\
\text { Percentage (\%) }\end{array}$ \\
\hline $\boldsymbol{k}$ & $l$ & Input & Output & \\
\hline 1 & 0 & $v(t)$ & - & 100 \\
\hline 2 & 0 & $v(t), d v(t) / d t$ & - & 9.79 \\
\hline 2 & 2 & $v(t), d v(t) / d t$ & $d i(t) / d t$ & 1.6 \\
\hline
\end{tabular}

looping trajectory $i(t)-v(t)$ of the studied MD is shown in Fig. 3, which reveals that the $i(t)$ is not a single-valued function of $v(t)$. Figures 4 to 6 illustrate the trajectory of $i(t)$

as a function of $\{v(t), d v(t) / d t\},\{d v(t) / d t, d i(t) / d t\}$, and $\{v(t), d i(t) / d t\}$ spaces. As it can be seen with all the possible combinations of the three dimensional embedding variables, it was not possible to obtain a single-valued mapping function to the output current. Therefore, we will use the FNN algorithm to increase the order of $Z_{k, l}(t)$ until a single-valued function is obtained.

A large time series data with 6001 samples is recorded for 8 periods of the sinusoidal excitation. The sampling time $T_{S}$ is equal to $1.1 \mathrm{~ms}$. This recorded $\mathrm{I} / \mathrm{O}$ data set will form the regression vector (2) or (3) that will be used for the FNN algorithm, whose results revealed to be relatively unchanged over a wide range of threshold values of $R$ [7]. A threshold of $R=12$ was applied to the entire time series. The variation of percentage of the FNN as a function of the embedding inputoutput dimensions is shown in Fig. 7 and the numerical results are reported in Table I. The percentage of FNN is an indication to check whether a single-valued function can be obtained for the selected state space dimensionality by varying the input space dimension $(k)$ and the output space dimension $(l)$, by adding more delayed versions ( i.e. $\tau=T / 4$ ) of $v(t)$ or $i(t)$, respectively.

As seen from Fig.7, the percentage of FNN drops to a low value of $1.6 \%$ when the regression vector takes the form $\{v(t), d v(t) / d t, d i(t) / d t\}$. This result was achieved by using the embedding variables $\{v(t), d i(t) / d t\}($ e.g. $l=2)$ and varying the input space $k$ to converge to the FNN value of $1.6 \%$ for $k=2$ as shown by the blue solid line in Fig.7. The same result was also obtained by fixing the embedding variables $\{v(t), d v(t) / d t\}$ (e.g. $k=2)$ and varying the output space $l$ to converge to $1.6 \%$ for $l=2$ as depicted by dashed red line. Consequently, the input/output dynamics of the system can be represented well by a reduced system with only two embedding variables. Discarding the output space from the regression vector (i.e. $l=0$ ) will not reduce the percentage of FNN below $9.79 \%$ for $k \geq 4$.

\section{CONCLUSION}

This paper presented the identification of the dynamical statespace embedding variables based on the FNN technique. The proper delay of the regression vector is determined to obtain de-correlated input and output space signals that reflects the MD's nonlinear memory effects. The FNN algorithm then determines the number of the state-variables required to embed the dynamics of the MD based on the acquired input-output time-domain signals and delayed values. The numerical results of the FNN processing demonstrates that the embedding variables $\{v(t), d v(t) / d t, d i(t) / d t\}$ was appropriate to embed the dynamics of the MD under test and to achieve a singlevalued input-output multivariate mapping between the device outputs as a function of the state variables. The achieved result can be further processed to generate a behavioral model based on polynomial or artificial neural network curve fitting in orde to compare the results to the HP model.

\section{ACKNOWLEDGMENT}

The described research was performed in laboratories supported FCT through the grant UID/EEA/50008/2013. Authors Wael Dghais and Jonathan Rodriguez would like to acknowledge funding from the Fundação para a Ciência e Tecnologia and the ENIAC JU (THINGS2DO-GA n. 621221 ENIAC-9/0001/2013)

\section{REFERENCES}

[1] L. Chua, S. M. Kang, "Memristive devices and systems", Proc. IEEE pp. 209 - 223vol. 64, no. 2, 1976

[2] Adhikaru AP, Sah MP, Kim H, Chua L. The fingerprints of memristor. IEEE Trans Circuits Systems, vol.60, no.11, pp. 3008 - 30212013.

[3] D. Schreurs, J. Wood, N. B. Tufillaro, L. Barford, D. E. Root, "Construction of behavioral models for microwave devices from timedomain large signal measurements to speed up high-level desig simulations", J. RF Microwave Computer-Aided Eng., vol. 13, no. 2003.

[4] M. D. Pickett, D. B. Strukov, J. L. Borghetti, J. J. Yang, G. S. Snider, D. R. Stewart, R. S. Williams, "Switching dynamics in titaniumdioxide memristive devices", J. Appl. Phys., vol. 106, no. 7, 2009

[5] A. Ascoli, F. Corinto, V. Senger, R. Tetzlaff, "Memristor model comparison", IEEE Circuits Syst. Mag., vol. 13, no. 2, 2013

[6] M.B. Kennel, R. Brown, H.D.I. Abarbanel, "Determining embedding dimension for phase-space reconstruction using a geometric construction", Physical Review A, pp. 3403-3411 vol 45, no.6, 1992

[7] C. Rhodes, M. Morari, "Determining the model order of nonlinea input/output systems", AIChE Journal, pp. 151-163, vol, 44, no.11998.

8] J. Wood, D. E. Root, N. B. Tuffilaro,"A behavioral modeling approach to nonlinear model-order reduction for $\mathrm{RF} /$ microwave ICs an systems", IEEE Trans. Microw. Theory Tech., pp. 2274 - 2284, vol. 52 , no. 9,2004

[9] T. Prodromakis, B. Peh , C. Papavassiliou, C. Toumazou, "A versatile memristor model with nonlinear dopant kinetics", IEEE Trans. Electron. Devices, vol. 58, no. $9,2011$.
Commented [n1]: This means the same FFN percentage for two different embedding, is this what you mean? If so, you then select the embedding space with lower dimension to model the device. Also, as far as I can remember we were discussing why the FNN percentage never drop to zero. Do you have an answer to this?
Also, if you have multiple representations an none is directly reproducing the dynamics of the state in equation (1), what can we conclude? 